\title{
ON A CATEGORY OF COTANGENT SUMS RELATED TO THE NYMAN-BEURLING CRITERION FOR THE RIEMANN HYPOTHESIS
}

\author{
DEREVYANKO NIKITA AND KOVALENKO KIRILL
}

\begin{abstract}
The purpose of the present paper is to provide a general overview of a variety of results related to a category of cotangent sums which have been proven to be associated to the so-called Nyman-Beurling criterion for the Riemann Hypothesis. These sums are also related to the Estermann Zeta function.
\end{abstract}

\section{INTRODUCTION}

This paper is focused on applications of certain cotangent sums to different problems related to the Riemann Hypothesis. The expression for the sums in question is the following

\section{Definition 1.1.}

$$
c_{0}\left(\frac{r}{b}\right):=-\sum_{m=1}^{b-1} \frac{m}{b} \cot \left(\frac{\pi m r}{b}\right),
$$

where $r, b \in \mathbb{N}, b \geq 2,1 \leq r \leq b$ and $(r, b)=1$.

1.1. Nyman-Beurling criterion for the Riemann Hypothesis. There are many interesting results concerning these cotangent sums, but initially we will present some general information about the Riemann Hypothesis and some related problems. Moreover, our aim is to provide motivation for the use of cotangent sums in these problems.

In this paper we shall denote a complex variable by $s=\sigma+i t$, where $\sigma$ and $t$ are the real and imaginary part of $s$ respectively.

Definition 1.2. The Riemann zeta function is a function of the complex variable s defined in the half-plane $\{\sigma>1\}$ by the absolutely convergent series

$$
\zeta(s):=\sum_{n=1}^{\infty} \frac{1}{n^{s}}
$$

As shown by B. Riemann, $\zeta(s)$ extends to $\mathbb{C}$ as a meromorphic function with only a simple pole at $s=1$, with the residue 1 , and satisfies the functional equation

$$
\zeta(s)=2^{s} \pi^{s-1} \sin \left(\frac{\pi s}{2}\right) \Gamma(1-s) \zeta(1-s) .
$$

For negative integers, one has a convenient representation of the Riemann zeta function in terms of Bernoulli numbers:

$$
\zeta(-n)=(-1)^{n} \frac{B_{n+1}}{n+1}, \text { for } n \geq 0 .
$$

By the above formula one can easily deduce that $\zeta(s)$ vanishes when $s$ is a negative even integer because $B_{m}=0$ for all odd $m$ other than 1 . The negative even integers are called 
trivial zeros of the Riemann zeta function. All other complex points where $\zeta(s)$ vanishes are called non-trivial zeros of the Riemann zeta function, and they play a significant role in the distribution of primes.

The actual connection with the distribution of prime numbers was observed in Riemann's 1859 paper. It is in this paper that Riemann proposed his well known hypothesis.

Hypothesis 1.1 (Riemann). The Riemann zeta function $\zeta(s)$ attains its non-trivial zeros only in complex points with $\sigma=\frac{1}{2}$. The line on the complex plane given by the equation $\sigma=\frac{1}{2}$ is usually called "critical".

The Nyman-Beurling-Baez-Duarte-Vasyunin (also simply known as Nyman-Beurling) approach to the Riemann Hypothesis enables us to associate the study of cotangent sums and the Riemann Hypothesis through the following theorem:

Theorem 1.1. The Riemann Hypothesis is true if and only if

$$
\lim _{N \rightarrow+\infty} d_{N}=0
$$

where

$$
d_{N}^{2}=\inf _{D_{N}} \frac{1}{2 \pi} \int_{-\infty}^{+\infty}\left|1-\zeta\left(\frac{1}{2}+i t\right) D_{N}\left(\frac{1}{2}+i t\right)\right|^{2} \frac{d t}{\frac{1}{4}+t^{2}}
$$

and the infimum is taken over all Dirichlet polynomials

$$
D_{N}(s)=\sum_{n=1}^{N} \frac{a_{n}}{n^{s}}, a_{n} \in \mathbb{C} .
$$

In his paper [2], B. Bagchi used a slightly different formulation of Theorem 1.1. In order to state it, we have to introduce some definitions.

Definition 1.3. The Hardy space $H^{2}(\Omega)$ is the Hilbert space of all analytic functions $F$ on the half-plane $\Omega$ (we define it for a right half-plane $\left\{\sigma>\sigma_{0}\right\}$ of the complex plane) such that

$$
\|F\|^{2}:=\sup _{\sigma>\sigma_{0}} \frac{1}{2 \pi} \int_{-\infty}^{+\infty}|F(\sigma+i t)|^{2} d t<\infty .
$$

Everywhere in this section we will use $\Omega=\left\{\sigma>\frac{1}{2}\right\}$.

Definition 1.4. For $0 \leq \lambda \leq 1$, let $F_{\lambda} \in H^{2}(\Omega)$ be defined by

$$
F_{\lambda}(s)=\left(\lambda^{s}-\lambda\right) \frac{\zeta(s)}{s}, s \in \Omega,
$$

and for $l=1,2,3, \ldots$, let $G_{l} \in H^{2}(\Omega)$ be defined by $G_{l}=F_{\frac{1}{l}}$, i.e

$$
G_{l}(s)=\left(l^{-s}-l^{-1}\right) \frac{\zeta(s)}{s}, s \in \Omega .
$$

Also, let $E \in H^{2}(\Omega)$ be defined by

$$
E(s)=\frac{1}{s}, s \in \Omega .
$$

Now we can state the reformulation of Theorem 1.1 which was used in paper [2]. 
Theorem 1.2. The following statements are equivalent:

(1) The Riemann Hypothesis is true;

(2) $E$ belongs to the closed linear span of the set $\left\{G_{l}: l=1,2,3 \ldots\right\}$;

(3) E belongs to the closed linear span of the set $\left\{F_{\lambda}: 0 \leq \lambda \leq 1\right\}$.

The plan of the proof is to verify three implications: $1 \rightarrow 2,2 \rightarrow 3$ and $3 \rightarrow 1$.

The first implication is the most challenging of all three. It is proven using some famous results obtained under the assumption that the Riemann Hypothesis is true, among which are Littlewood's theorem 1.3 and the Lindelöf hypothesis 1.2, and some standard techniques of functional analysis, particularly concerning convergence in the norm. More details can be found in the original paper by B. Bagchi [2].

Hypothesis 1.2 (Lindelöf). If the Riemann Hypothesis is true, then

$$
\forall \varepsilon>0, \zeta\left(\frac{1}{2}+i t\right)=O\left(t^{\varepsilon}\right)
$$

Remark: A very interesting and novel approach to the Lindelöf hypothesis is presented by A. Fokas [12].

Theorem 1.3 (Littlewood). If the following conditions are satisfied:

- $\lim _{r \rightarrow 1^{-}} \sum_{n=0}^{\infty} r^{n} c_{n}=a, \forall i c_{i} \in \mathbb{C}, a \in \mathbb{C}$,

- $c_{n}=O\left(\frac{1}{n}\right)$,

then

$$
\sum_{n=0}^{\infty} c_{n}=a
$$

The second implication follows from the embedding

$$
\left\{G_{l}: l=1,2,3 \ldots\right\} \subset\left\{F_{\lambda}: 0 \leq \lambda \leq 1\right\} .
$$

To prove the third implication $(3 \rightarrow 1)$, suppose that the Riemann Hypothesis is false. Then $\exists s_{0}=\sigma_{0}+i t_{0}: \zeta\left(s_{0}\right)=0$ and $\sigma_{0} \neq \frac{1}{2}$, which implies that $\forall 0 \leq \lambda \leq 1 F_{\lambda}\left(s_{0}\right)=0$. That together with statement 3 gives that $E\left(s_{0}\right)=\frac{1}{s_{0}}=0$, i.e $0=1$. This contradiction completes the proof.

1.2. The cotangent sum's applications to problems related to the Riemann Hypothesis. The main motivation behind the study of the cotangent sum (1) follows from Theorem 1.1, which constitutes an equivalent form of the Riemann Hypothesis.

Asymptotics for $d_{N}$ (4) under the assumption that the Riemann Hypothesis is true have been studied in several papers. S. Bettin, J. Conrey and D. Farmer in [9] obtained the following result.

Theorem 1.4. If the Riemann Hypothesis is true and if

$$
\sum_{|\operatorname{Im}(\rho)| \leq T} \frac{1}{\left|\zeta^{\prime}(\rho)\right|^{2}} \ll T^{\frac{3}{2}-\delta}
$$


for some $\delta>0$, with the sum on the left hand side taken over all distinct zeros $\rho$ of the Riemann zeta function with imaginery part less than or equal to $T$, then

$$
\frac{1}{2 \pi} \int_{-\infty}^{+\infty}\left|1-\zeta\left(\frac{1}{2}+i t\right) V_{N}\left(\frac{1}{2}+i t\right)\right|^{2} \frac{d t}{\frac{1}{4}+t^{2}} \sim \frac{2+\gamma-\log 4 \pi}{\log N}
$$

for

$$
V_{N}(s):=\sum_{n=1}^{N}\left(1-\frac{\log n}{\log N}\right) \frac{\mu(n)}{n^{s}} .
$$

We should mention that in the sequel $\gamma$ stands for the Euler-Mascheroni constant. Also, here $\mu$ is the Möbius function.

Also, from results of [9] it follows that under some restrictions, the infimum from (4) is attained for $D_{N}=V_{N}$.

Nevertheless, it is interesting to obtain an unconditional estimate for $d_{N}$.

In order to proceed further, we shall study equation (4) in more detail. In particular, we can expand the square in the integral:

$$
\begin{aligned}
d_{N}= & \inf _{D_{N}}\left(\int_{-\infty}^{+\infty}\left(1-\zeta\left(\frac{1}{2}+i t\right) D_{N}\left(\frac{1}{2}+i t\right)-\zeta\left(\frac{1}{2}-i t\right) \overline{D_{N}}\left(\frac{1}{2}+i t\right)\right) \frac{d t}{\frac{1}{4}+t^{2}}\right. \\
& \left.+\int_{-\infty}^{+\infty}\left|\zeta\left(\frac{1}{2}+i t\right)\right|^{2}\left|D_{N}\left(\frac{1}{2}+i t\right)\right|^{2} \frac{d t}{\frac{1}{4}+t^{2}}\right) .
\end{aligned}
$$

The integral in the second summand can be expressed as

$$
\sum_{1 \leq r, b \leq N} a_{r} \overline{a_{b}} r^{-\frac{1}{2}} b^{-\frac{1}{2}} \int_{-\infty}^{+\infty}\left|\zeta\left(\frac{1}{2}+i t\right)\right|^{2}\left(\frac{r}{b}\right)^{i t} \frac{d t}{\frac{1}{4}+t^{2}}
$$

where $a_{i}$ are from the definition (5) of $D_{N}$.

Therefore, the integral

$$
\int_{-\infty}^{\infty}\left|\zeta\left(\frac{1}{2}+i t\right)\right|^{2}\left(\frac{r}{b}\right)^{i t} \frac{d t}{\frac{1}{4}+t^{2}}
$$

plays an important role in the Nyman-Beurling criterion for the Riemann Hypothesis. Moreover, one can prove that this integral can be expressed via the so-called Vasyunin sum.

Definition 1.5. The Vasyunin sum is defined as follows:

$$
V\left(\frac{r}{b}\right):=\sum_{m=1}^{b-1}\left\{\frac{m r}{b}\right\} \cot \left(\frac{\pi m r}{b}\right),
$$

where $\{x\}=x-\lfloor x\rfloor, x \in \mathbb{R}$.

The following proposition holds true: 


\section{Proposition 1.1.}

$$
\begin{aligned}
& \frac{1}{2 \pi(r b)^{1 / 2}} \int_{-\infty}^{\infty}\left|\zeta\left(\frac{1}{2}+i t\right)\right|^{2}\left(\frac{r}{b}\right)^{i t} \frac{d t}{\frac{1}{4}+t^{2}} \\
& \quad=\frac{\log 2 \pi-\gamma}{2}\left(\frac{1}{r}+\frac{1}{b}\right)+\frac{b-r}{2 r b} \log \frac{r}{b}-\frac{\pi}{2 r b}\left(V\left(\frac{r}{b}\right)+V\left(\frac{b}{r}\right)\right) .
\end{aligned}
$$

One can note that the only non-explicit function on the right hand side of this formula is the Vasyunin sum.

The next equation connects this result with the cotangent sums in question.

Proposition 1.2. It holds that

$$
V\left(\frac{r}{b}\right)=-c_{0}\left(\frac{\bar{r}}{b}\right),
$$

where $\bar{r}$ is such that $\bar{r} r \equiv 1(\bmod b)$.

The cotangent sum $c_{0}$ can also be used to describe some special values of the Estermann zeta function.

Definition 1.6. The Estermann zeta function $E\left(s, \frac{r}{b}, \alpha\right)$ is defined by the Dirichlet series

$$
E\left(s, \frac{r}{b}, \alpha\right)=\sum_{n \geq 1} \frac{\sigma_{\alpha}(n) \exp \left(\frac{2 \pi i n r}{b}\right)}{n^{s}},
$$

where Re $s>\operatorname{Re} \alpha+1, b \geq 1,(r, b)=1$, and

$$
\sigma_{\alpha}(n)=\sum_{d \mid n} d^{\alpha}
$$

One can show that the Estermann zeta function $E\left(s, \frac{r}{b}, \alpha\right)$ satisfies the following functional equation:

$$
\begin{aligned}
& E\left(s, \frac{r}{b}, \alpha\right)=\frac{1}{\pi}\left(\frac{b}{2 \pi}\right)^{1+\alpha-2 s} \Gamma(1-s) \Gamma(1+\alpha-s) \\
& \quad \times\left(\cos \left(\frac{\pi \alpha}{2}\right) E\left(1+\alpha-s, \frac{\bar{r}}{b}, \alpha\right)-\cos \left(\pi s-\frac{\pi \alpha}{2}\right) E\left(1+\alpha-s,-\frac{\bar{r}}{b}, \alpha\right)\right),
\end{aligned}
$$

where $r$ is such that $\bar{r} r \equiv 1(\bmod b)$.

Properties of $E\left(0, \frac{r}{b}, 0\right)$ were used by R. Balasubramanian, J. Conrey, and D. HeathBrown [3] to prove an asymptotic formula for

$$
I=\int_{0}^{T}\left|\zeta\left(\frac{1}{2}+i t\right)\right|^{2}\left|A\left(\frac{1}{2}+i t\right)\right|^{2} d t
$$

where $A(s)$ is a Dirichlet polynomial.

Asymptotic results for the function $I$ as well as other functions of this type, are useful for estimating a lower bound for the portion of zeros of the Riemann zeta function $\zeta(s)$ on the critical line.

The following result of M. Ishibashi from [14] concerning $E\left(s, \frac{r}{b}, \alpha\right)$ for $s=0$, provides the connection of the Estermann zeta function with the cotangent sum $c_{0}\left(\frac{r}{b}\right)$, simply by setting $\alpha=0$. 
Theorem 1.5 (Ishibashi). Let $b \geq 2,1 \leq r \leq b,(r, b)=1, \alpha \in \mathbb{N} \cup\{0\}$. Then for even $\alpha$, it holds that

$$
E\left(0, \frac{r}{b}, \alpha\right)=\left(-\frac{i}{2}\right)^{\alpha+1} \sum_{m=1}^{b-1} \frac{m}{b} \cot ^{(\alpha)}\left(\frac{\pi m r}{b}\right)+\frac{1}{4} \delta_{\alpha, 0},
$$

where $\delta_{\alpha, 0}$ is the Kronecker delta function.

For odd $\alpha$, it holds that

$$
E\left(0, \frac{r}{b}, \alpha\right)=\frac{B_{\alpha+1}}{2(\alpha+1)} .
$$

In the special case when $r=b=1$, we have

$$
E(0,1, \alpha)=\frac{(-1)^{\alpha+1} B_{\alpha+1}}{2(\alpha+1)},
$$

where $B_{m}$ is the $m$-th Bernoulli number and $B_{2 m+1}=0$,

$$
B_{2 m}=\frac{(-1)^{m+1} 2(2 m) !}{(2 \pi)^{2 m}} \zeta(2 m), \text { for } m \geq 1 .
$$

Thus for $b \geq 2,1 \leq r \leq b,(r, b)=1$, it follows that

$$
E\left(0, \frac{r}{b}, 0\right)=\frac{1}{4}+\frac{i}{2} c_{0}\left(\frac{r}{b}\right),
$$

where $c_{0}\left(\frac{r}{b}\right)$ is our cotangent sum from (1).

\section{Central properties of the cotangent sum $c_{0}$}

Now we can state some crucial results concerning the cotangent sum $c_{0}$. In [23], M. Th. Rassias proved the following asymptotic formula:

Theorem 2.1. For $b \geq 2, b \in \mathbb{N}$, we have

$$
c_{0}\left(\frac{1}{b}\right)=\frac{1}{\pi} b \log b-\frac{b}{\pi}(\log 2 \pi-\gamma)+O(1) .
$$

Subsequently in [19], M. Th. Rassias and H. Maier established an improvement, or rather an asymptotic expansion, of Theorem 2.1.

Theorem 2.2. Let $b, n \in \mathbb{N}, b \geq 6 N$, with $N=\left\lfloor\frac{n}{2}\right\rfloor+1$. There exist absolute real constants $A_{1}, A_{2} \geq 1$ and absolute real constants $E_{l}$, where $l \in \mathbb{N}$, with $\left|E_{l}\right| \leq\left(A_{1} l\right)^{2 l}$, such that for each $n \in \mathbb{N}$ we have

$$
c_{0}\left(\frac{1}{b}\right)=\frac{1}{\pi} b \log b-\frac{b}{\pi}(\log 2 \pi-\gamma)-\frac{1}{\pi}+\sum_{l=1}^{n} E_{l} b^{-l}+R_{n}^{*}(b),
$$

where

$$
\left|R_{n}^{*}\right| \leq\left(A_{2} n\right)^{4 n} b^{-(n-1)} .
$$

It is essential that both of these results were obtained using a common underlying idea proposed in [23]. First of all, one can obtain the following relation between sums of cotangents and sums with fractional parts: 


$$
\sum_{\substack{a \geq 1 \\ b \nmid a}} \frac{b(1-2\{a / b\})}{a}=\sum_{\substack{a \geq 1 \\ b \nmid a}} \sum_{m=1}^{b} \cot \left(\frac{\pi m}{b}\right) \frac{\sin \left(\frac{2 \pi m}{b} a\right)}{a} .
$$

This relation provided the following proposition:

Proposition 2.1. For every positive integer $b \geq 2$, we have

$$
c_{0}\left(\frac{1}{b}\right)=\sum_{\substack{a \geq 1 \\ b \nmid a}} \frac{b(1-2\{a / b\})}{a} .
$$

Then the difficulty lies in obtaining a good approximation of the sum $S(L ; b)$ defined by

$$
S(L ; b):=2 b \sum_{1 \leq a \leq L} \frac{1}{a}\left\lfloor\frac{a}{b}\right\rfloor .
$$

The difference between the estimates from Theorems 2.1 and 2.2 is that stronger approximation techniques were applied in [19] to obtain more information about $S(L ; b)$. Namely, the generalized Euler summation formula (23) was used to improve the result of Theorem 2.1 .

Definition 2.1. If $f$ is a function that is differentiable at least $(2 N+1)$ times in $[0, Z]$, let

$$
r_{N}(f, Z)=\frac{1}{(2 N+1) !} \int_{0}^{Z}(u-\lfloor u\rfloor+B)^{(2 N+1)} f^{(2 N+1)}(u) d u,
$$

with the following notation:

$$
(u-\lfloor u\rfloor+B)^{(2 N+1)}=((u-\lfloor u\rfloor)+B)^{(2 N+1)}:=\sum_{j=0}^{2 N+1}\left(\begin{array}{c}
2 N+1 \\
j
\end{array}\right)(u-\lfloor u\rfloor)^{j} B_{2 N+1-j},
$$

where $B_{2 j}$ are the Bernoulli numbers.

Theorem 2.3 (Generalized Euler summation formula). Let $f$ be $(2 N+1)$ times differentiable in the interval $[0, Z]$. Then

$$
\begin{aligned}
& \sum_{\nu=0}^{Z} f(\nu)=\frac{f(0)+f(Z)}{2}+\int_{0}^{Z} f(u) d u \\
& \quad+\sum_{j=1}^{N} \frac{B_{2 j}}{(2 j) !}\left(f^{(2 j-1)}(Z)-f^{(2 j-1)}(0)\right)+r_{N}(f, Z) .
\end{aligned}
$$

Particularly, H. Maier and M. Th. Rassias used Theorem 2.3 to obtain the following new representation for $S(L ; b)$.

Theorem 2.4. For $N \in \mathbb{N}$, we have

$$
\begin{gathered}
S(L ; b)=2 b \sum_{k \leq L / b} k\left(\log \frac{(k+1) b-1}{k b-1}+\frac{1}{2} F_{1}(k, b)\right) \\
+2 b \sum_{j=1}^{N} \frac{B_{2 j}}{2 j} \sum_{k \leq L / b} k F_{2 j}(k, b)+2 b r_{N}\left(f, \frac{L}{b}\right), \\
7
\end{gathered}
$$


where the function $f$ satisfies

$$
f(u)=\left\{\begin{array}{l}
\frac{1}{u}, \text { if } u \geq 1 \\
0, \text { if } u=0
\end{array}\right.
$$

and $f \in C^{\infty}([0, \infty))$ with $f^{(j)}(0)=0$ for $j \leq 2 N+1$.

The new form of $S(L ; b)$ from (24) lead essentially to the proof of Theorem 2.2.

Furthermore, H. Maier and M. Th. Rassias obtained even more interesting results concerning $c_{0}\left(\frac{r}{b}\right)$ for a fixed arbitrary positive integer value of $r$ and for large integer values of $b$, which give us a deeper understanding of our cotangent sum for almost all values of $r$ and $b$.

Proposition 2.2. For $r, b \in \mathbb{N}$ with $(r, b)=1$, it holds that

$$
c_{0}\left(\frac{r}{b}\right)=\frac{1}{r} c_{0}\left(\frac{1}{b}\right)-\frac{1}{r} Q\left(\frac{r}{b}\right),
$$

where

$$
Q\left(\frac{r}{b}\right)=\sum_{m=1}^{b-1} \cot \left(\frac{\pi m r}{b}\right)\left\lfloor\frac{r m}{b}\right\rfloor .
$$

Theorem 2.5. Let $r, b_{0} \in \mathbb{N}$ be fixed, with $\left(b_{0}, r\right)=1$. Let $b$ denote a positive integer with $b \equiv b_{0}(\bmod r)$. Then, there exists a constant $C_{1}=C_{1}\left(r, b_{0}\right)$, with $C_{1}\left(1, b_{0}\right)=0$, such that

$$
c_{0}\left(\frac{r}{b}\right)=\frac{1}{\pi r} b \log b-\frac{b}{\pi r}(\log 2 \pi-\gamma)+C_{1} b+O(1)
$$

for large integer values of $b$.

The function $c_{0}$ is also thoroughly studied in the papers of S. Bettin and J. Conrey $[7,8]$, where they have established a reciprocity formula for it. However, before we state the formula itself, we must give several definitions.

For $a \in \mathbb{C}$ and $\operatorname{Im}(s)>0$, consider

$$
\begin{gathered}
\mathscr{S}_{a}(s):=\sum_{n=1}^{\infty} \sigma_{a}(n) e(n s) e^{2 \pi i n s}, \\
E_{a}(s):=1+\frac{2}{\zeta(-a)} \mathscr{S}_{a}(s) .
\end{gathered}
$$

It is worth mentioning that for $a=2 k+1, k \in \mathbb{Z}_{\geq 1}, E_{a}$ is the well known Eisenstein series of weight $2 k+2$.

Definition 2.2. For $a \in \mathbb{C}$ and $\operatorname{Im}(s)>0$, define the function

$$
\psi_{a}(s):=E_{a+1}(s)-\frac{1}{s^{a+1}} E_{a+1}\left(-\frac{1}{s}\right) .
$$

For $a=2 k, k \geq 2$, the function $\psi_{a}(s)$ is equal to zero, because of the modularity property of the Eisenstein series. Unfortunately, it is not true for other values of $a$, but the functions $\psi_{a}(s)$ have some remarkable properties, which were described in detail by S. Bettin and J. Conrey.

Now we can state the theorem proven in paper [7]. 
Theorem 2.6. The function $c_{0}$ satisfies the following reciprocity formula:

$$
c_{0}\left(\frac{r}{b}\right)+\frac{b}{r} c_{0}\left(\frac{b}{r}\right)-\frac{1}{2 \pi r}=\frac{i}{2} \psi_{0}\left(\frac{r}{b}\right) .
$$

This result implies that the value of $c_{0}\left(\frac{r}{b}\right)$ can be computed within a prescribed accuracy in a polynomial of $\log b$.

S. Bettin and J. Conrey highlighted that the reciprocity formula from 2.6 is very similar to that of the Dedekind sum 5.1. We will consider Dedekind sums in more detail in section 5 of this paper.

In [8] the result for $c_{0}$ was generalized for the sums

$$
c_{a}\left(\frac{r}{b}\right):=b^{a} \sum_{m=1}^{b-1} \cot \left(\frac{\pi m r}{b}\right) \zeta\left(-a, \frac{m}{b}\right),
$$

where $\zeta(s, x)$ is the Hurwitz zeta function.

2.1. Ellipse. It is interesting to mention that if one examines the graph of $c_{0}\left(\frac{r}{b}\right)$ for hundreds of integer values of $b$ by the use of MATLAB, the resulting figures 1 and 2 always have a shape similar to an ellipse.

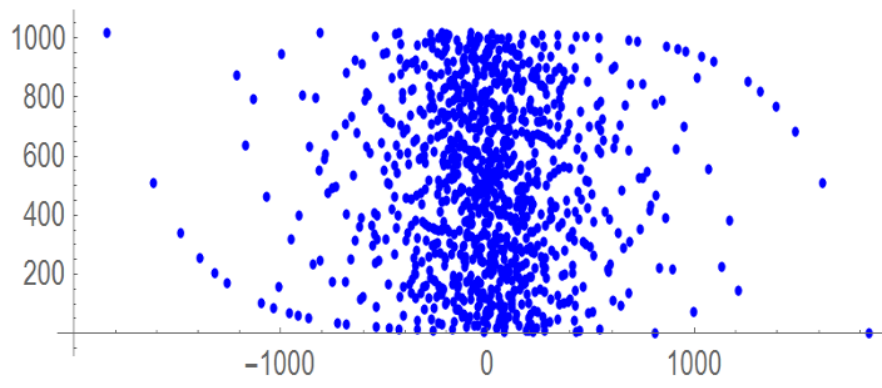

FIG 1 - axis $O x: r$, axis $O y: c_{0}, b=1021$

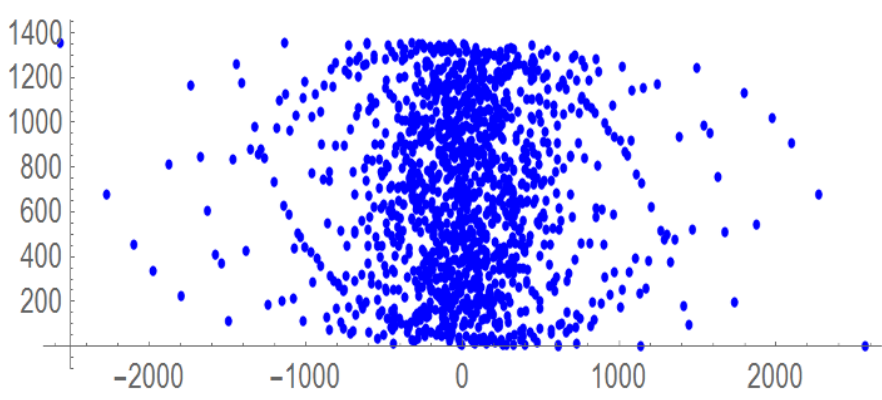

FIG 2 - axis $O x: r$, axis $O y: c_{0}, b=1357$

In [19] H. Maier and M. Th. Rassias tried to explain this phenomenon and obtained an important result, which establishes the equidistribution of certain normalized cotangent sums with respect to a positive measure. This is presented in the following theorem. 
Definition 2.3. For $z \in \mathbb{R}$, let

$$
F(z)=\operatorname{meas}\{x \in[0,1]: g(x) \leq z\},
$$

where "meas" denotes the Lebesgue measure,

$$
g(x):=\sum_{l=1}^{+\infty} \frac{1-2\{l x\}}{l},
$$

and

$C_{0}(\mathbb{R})=\{f \in C(\mathbb{R}): \forall \varepsilon>0, \exists$ a compact set $\mathcal{K} \subset \mathbb{R}$, such that $|f(x)|<\varepsilon, \forall x \notin \mathcal{K}\}$.

Remark. The convergence of the above series has been investigated by R.Bretèche and G.Tenenbaum (see Theorem 4.2). It depends on the partial fraction expansion of the number $x$.

Theorem 2.7. (i) $F$ is a continuous function of $z$.

(ii) Let $A_{0}, A_{1}$ be fixed constants, such that $1 / 2<A_{0}<A_{1}<1$. Let also

$$
H_{k}=\int_{0}^{1}\left(\frac{g(x)}{\pi}\right)^{2 k} d x
$$

so $H_{k}$ is a positive constant depending only on $k, k \in \mathbb{N}$.

There is a unique positive measure $\mu$ on $\mathbb{R}$ with the following properties:

(a) For $\alpha<\beta \in \mathbb{R}$ we have

$$
\mu([\alpha, \beta])=\left(A_{1}-A_{0}\right)(F(\beta)-F(\alpha)) .
$$

(b)

$$
\int x^{k} d \mu= \begin{cases}\left(A_{1}-A_{0}\right) H_{k / 2}, & \text { for even } k \\ 0, & \text { otherwise. }\end{cases}
$$

(c) For all $f \in C_{0}(\mathbb{R})$, we have

$$
\lim _{b \rightarrow+\infty} \frac{1}{\phi(b)} \sum_{\substack{r:(r, b)=1, A_{0} b \leq r \leq A_{1} b}} f\left(\frac{1}{b} c_{0}\left(\frac{r}{b}\right)\right)=\int f d \mu,
$$

where $\phi(\cdot)$ denotes the Euler phi-function.

\section{Outline of the proof.}

In [19] H. Maier and M. Th. Rassias proved Theorem 2.2, which constitutes an improvement of their earlier Theorem 2.1.

Additionally, they investigated the cotangent sum $c_{0}\left(\frac{r}{b}\right)$ for a fixed arbitrary positive integer value of $r$ and for large integer values of $b$ and proved Theorem 2.5 as well as the following results

Theorem 2.8. Let $k \in \mathbb{N}$ be fixed. Let also $A_{0}, A_{1}$ be fixed constants such that $\frac{1}{2}<A_{0}<A_{1}<1$. Then there exists a constant $E_{k}>0$, depending only on $k$, such that (a) 


$$
\sum_{\substack{r:(r, b)=1 \\ A_{0} b \leq r \leq A_{1} b}} Q\left(\frac{r}{b}\right)^{2 k}=E_{k}\left(A_{1}^{2 k+1}-A_{0}^{2 k+1}\right) b^{4 k} \phi(b)(1+o(1)) \quad(b \rightarrow+\infty),
$$

(b)

$$
\sum_{\substack{r:(r, b)=1 \\ A_{0} b \leq r \leq A_{1} b}} Q\left(\frac{r}{b}\right)^{2 k-1}=o\left(b^{4 k-2} \phi(b)\right) \quad(b \rightarrow+\infty),
$$

(c)

$$
\sum_{\substack{r:(r, b)=1 \\ A_{0} b \leq r \leq A_{1} b}} c_{0}\left(\frac{r}{b}\right)^{2 k}=H_{k}\left(A_{1}-A_{0}\right) b^{2 k} \phi(b)(1+o(1)) \quad(b \rightarrow+\infty),
$$

(d)

$$
\sum_{\substack{r:(r, b)=1 \\ A_{0} b \leq r \leq A_{1} b}} c_{0}\left(\frac{r}{b}\right)^{2 k-1}=o\left(b^{2 k-1} \phi(b)\right) \quad(b \rightarrow+\infty),
$$

with

$$
E_{k}=\frac{H_{k}}{(2 k+1)}
$$

Using the method of moments, one can deduce detailed information about the distribution of the values of $c_{0}\left(\frac{r}{b}\right)$, where $A_{0} b \leq r \leq A_{1} b$ and $b \rightarrow+\infty$. Namely, one can prove Theorem 2.7.

\section{The MAXIMUM of $c_{0}$ IN RATIONAL NUMBERS IN SHORT INTERVALS}

In this section we consider some results about the maximum of $c_{0}$ in rational numbers in short intervals. More precicely, consider the following definition:

Definition 3.1. Let $0<A_{0}<1,0<C<1 / 2$. For $b \in \mathbb{N}$ we set

$$
\Delta:=\Delta(b, C)=b^{-C} .
$$

We define

$$
M\left(b, C, A_{0}\right):=\max _{A_{0} b \leq r<\left(A_{0}+\Delta\right) b}\left|c_{0}\left(\frac{r}{b}\right)\right| .
$$

In [20] H. Maier and M. Th. Rassias proved the following theorems.

Theorem 3.1. Let $D$ satisfy $0<D<\frac{1}{2}-C$. Then we have for sufficiently large $b$ :

$$
M\left(b, C, A_{0}\right) \geq \frac{D}{\pi} b \log b .
$$

An important part of the proof is the following key proposition. 
Proposition 3.1. Let $\left\langle a_{0} ; a_{1}, a_{2}, \ldots, a_{n}\right\rangle$ be the continued fraction expansion of $\frac{\bar{r}}{b} \in \mathbb{Q}$. Moreover, let $\frac{u_{l}}{v_{l}}$ be the $l$-th partial quotient of $\frac{\bar{r}}{b}$. Then

$$
c_{0}\left(\frac{r}{b}\right)=-b \sum_{1 \leq l \leq n} \frac{(-1)^{l}}{v_{l}}\left(\left(\frac{1}{\pi v_{l}}\right)+\psi\left(\frac{v_{l-1}}{v_{l}}\right)\right) .
$$

Here $\psi$ is an analytic function satisfying

$$
\psi(x)=-\frac{\log (2 \pi x)-\gamma}{\pi x}+O(\log x),(x \rightarrow 0) .
$$

The proposition was proven in [6] by S. Bettin.

Definition 3.2. Let $\Delta$ be as in Definition 3.1 and $\Omega>0$. We set

$$
N(b, \Delta, \Omega):=\#\left\{r: A_{0} b \leq r<\left(A_{0}+\Delta\right) b,|\bar{r}| \leq \Omega b\right\} .
$$

Another key proposition, proven in [20], is the following:

Proposition 3.2. Let $\varepsilon<0$ be such that

$$
D+\varepsilon<\frac{1}{2}-C
$$

Set

$$
\Omega:=b^{-(D+\varepsilon)} .
$$

Then for sufficiently large $b$ it holds

$$
N(b, \Delta, \Omega)>0 .
$$

Let $\left\{\frac{u_{i}}{v_{i}}\right\}_{i=1}^{s}$ be the sequence of partial fractions of such $\frac{\bar{r}}{b}$. From

we obtain

$$
\Omega \geq \frac{\bar{r}}{b} \geq \frac{1}{v_{1}+1}
$$

$$
v_{1}+1 \geq \Omega^{-1}
$$

Then by proposition 3.2 we have

$$
\sum_{l>1}\left(\left(\frac{1}{\pi v_{l}}\right)+\psi\left(\frac{v_{l-1}}{v_{l}}\right)\right)<2 \varepsilon \log b, \text { for } b \geq b_{0}(\varepsilon) .
$$

Therefore,

$$
\left|c_{0}\left(\frac{r}{b}\right)\right| \geq \frac{2}{\pi} \log \left(\Omega^{-1}(1+o(1))\right) \quad b \rightarrow+\infty .
$$

This proves Theorem 3.1.

Theorem 3.2. Let $C$ be as in Theorem 3.1 and let $D$ satisfy $0<D<2-C-E$, where $E \geq 0$ is a fixed constant. Let $B$ be sufficiently large. Then we have

$$
M\left(b, C, A_{0}\right) \leq \frac{D}{\pi} b \log b
$$

for all $b$ with $B \leq b<2 B$, with at most $B^{E}$ exceptions. 


\section{Proof.}

We will need the following proposition.

Proposition 3.3. Let $\varepsilon>0, B \geq B \varepsilon, B<b \leq 2 B$. For $1 \leq r<b,(r, b)=1$, let $\left\{\frac{u_{i}}{v_{i}}\right\}_{i=1}^{s}$ be the sequence of partial fractions of $\frac{\bar{r}}{b}$. Then there are at most 3 values of $l$ for which

$$
\frac{1}{v_{l}} \psi\left(\frac{v_{l-1}}{v_{l}}\right) \geq \log \log b
$$

and at most one value of $l$ for which

$$
\frac{1}{v_{l}} \psi\left(\frac{v_{l-1}}{v_{l}}\right) \geq \varepsilon \log b
$$

Proof. Let $l_{i}(i=1,2,3,4)$ be such that

$$
\frac{1}{v_{l}} \psi\left(\frac{v_{l-1}}{v_{l}}\right) \geq \log \log b
$$

Then we have

$$
\begin{gathered}
v_{l_{1}} \geq \log \log b, \quad v_{l_{2}} \geq \exp \left(v_{l_{1}}\right) \geq \log b, \\
v_{l_{3}} \geq \exp \left(v_{l_{2}}\right) \geq b, \quad v_{l_{4}} \geq \exp \left(v_{l_{3}}\right) \geq \exp (b),
\end{gathered}
$$

in contradiction to $v_{s} \leq b$.

In the same manner we obtain from $v_{l_{j}} \geq \varepsilon \log b, j=1,2$ :

$$
v_{s} \geq \exp \left(\exp \left((\log b)^{\varepsilon}\right)\right)>b .
$$

Assume $\varepsilon>0$ to be fixed but arbitrarily small, $Z>0$ fixed but arbitrarily large.

Definition 3.3. By Proposition 3.3 there is at most one value of $l$ for which

$$
\frac{1}{v_{l}} \psi\left(\frac{v_{l-1}}{v_{l}}\right) \geq \varepsilon \log b
$$

In case of the existence of $l$, we write

$$
u_{l-1}(r, b)=u_{l-1}
$$

and

$$
v_{l-1}(r, b)=v_{l-1} .
$$

Then for $s, t$ with $1 \leq s, t \leq Z,(s, t)=1$, and for fixed $\theta$ with $0<\theta<1$,

$$
\mathcal{F}(s, t):=\left\{(b, r, \bar{r}): B \leq b<2 B, A_{0} b \leq r \leq\left(A_{0}+\Delta\right) b,\left|\frac{\bar{r}}{b}-\frac{s}{t}\right| \leq \theta, r \bar{r} \equiv 1(\bmod b)\right\} .
$$

Now we can formulate a proposition.

\section{Proposition 3.4.}

$$
\sum_{B \leq b<2 B} N(b, \Delta, \Omega) \leq \sum_{1 \leq s, t \leq Z}|\mathcal{F}(s, t)|
$$


By Dirichlet's approximation theorem there is $\left(C_{0}, D_{0}\right) \in \mathbb{Z}^{2}$ with $1 \leq D_{0} \leq B^{2}$, $\left(C_{0}, D_{0}\right)=1$, such that

$$
\left|A_{0}^{-1}-\frac{C_{0}}{D_{0}}\right| \leq \frac{1}{D_{0} B^{2}}
$$

Let us estimate the cardinality of the set $\mathcal{F}(s, t)$.

From $A_{0} b \leq r \leq\left(A_{0}+\Delta\right) b$, the definition of $\bar{r}$, and (30), we obtain

$$
r \bar{r}=y\left(\frac{C_{0}}{D_{0}} r+\frac{u}{D_{0}}\right), \text { with } y \in \mathbb{Z},
$$

which after multiplication by $C_{0} D_{0}$ becomes

$$
\left(C_{0} y-D_{0} \bar{r}\right)\left(C_{0} r-u\right)=-D_{0}\left(C_{0}-\bar{r} u\right) .
$$

If $C_{0}-\bar{r} u \neq 0$, one can deduce from the well-known estimate for the number of divisors of an integer that for a given pair $(\bar{r}, u)$, there are at most $O\left(B^{\varepsilon}\right)$ pairs $(r, y)$ such that (32) holds.

There are at most $O\left(B^{\varepsilon}\right)$ pairs $(\bar{r}, u)$ such that $C_{0}-\bar{r} u=0$. Thus we obtain

$$
|\mathcal{F}(s, t)|=O\left(B^{2+2 \varepsilon-C} \theta\right) .
$$

From Proposition 3.4 and (33) we obtain for $\Omega=\theta B$

$$
\sum_{B \leq b<2 B} N(b, \Delta, \Omega)=O\left(B^{2+2 \varepsilon-C} \theta\right) .
$$

We now apply (34) with $\theta=B-D_{0}$, where

$$
D>D^{\prime}>2-C-E .
$$

If we choose $\varepsilon>0$ sufficiently small, then we conclude from (34) the following:

For all $b$ with $B \leq b<2 B$ we have, with at most $B^{E}$ exceptions:

$$
N\left(b, B^{1-C}, \Omega\right)=0 .
$$

Thus,

$$
\frac{1}{v_{l}} \psi\left(\frac{v_{l-1}}{v_{l}}\right) \leq \frac{D^{\prime}}{\pi v_{l-1}} b \log b(1+o(1)), \forall l \leq Z .
$$

The result of Theorem 3.2 follows now from Propositions 3.1 and 3.3.

\section{The Function $g(x)$ And moments of $c_{0}$}

There is an interesting connection between the cotangent sums $c_{0}$ and the function

$$
g(x):=\sum_{l=1}^{+\infty} \frac{1-2\{l x\}}{l},
$$

which, as we mentioned above, naturally appeared in the investigation of the moments of $c_{0}$ and of the sum $Q\left(\frac{r}{b}\right)$, which is related to $c_{0}$ by Proposition 2.2. To be precise, this series is related to $c_{0}$ by the important Theorem 2.8 . 
Later S. Bettin in his paper [6] extended the result of Theorem 2.8 and proved the following:

Theorem 4.1. Let $b \geq 1$ and $k \geq 0$. Then

$$
\frac{1}{\phi(b)} \sum_{\substack{r=1 \\(r, b)=1}}^{b} c_{0}\left(\frac{r}{b}\right)^{k}=H_{k} b^{k}+O_{\varepsilon}\left(b^{k-1+\varepsilon}(A k \log b)^{2 k}\right),
$$

for some absolute constant $A>0$ and any $\varepsilon>0$.

Moreover, if $0 \leq A_{0}<A_{1} \leq 1$, then we have

$$
\frac{1}{\phi(b)} \sum_{\substack{(r, b)=1 \\ A_{0}<\frac{r}{b}<A_{1}}} c_{0}\left(\frac{r}{b}\right)^{k}=\left(A_{1}-A_{0}\right) H_{k} b^{k}+O_{\varepsilon}\left(b^{k-\frac{1}{2}+\varepsilon}(A k \log b)^{2 k}\right) .
$$

The function $g(x)$ is interesting not only in connection to the study of the cotangent sums $c_{0}$, but also in its own right. For example, it is also studied in [11] by R. Bretèche and G. Tenenbaum.

Theorem 4.2. For each $x \in \mathbb{Q}$ the series $g(x)$ converges.

For $x \in \mathbb{R} \backslash \mathbb{Q}$, the series $g(x)$ converges if and only if the series

$$
\sum_{m \geq 1}(-1)^{m} \frac{\log q_{m+1}}{q_{m}}
$$

converges, where $\left(q_{m}\right)_{m \geq 1}$ denotes the sequence of partial denominators of the continued fraction expansion of $x$.

Proof. The statement of the theorem is part of Theorem 4.4 of the paper by R. Bretèche and G. Tenenbaum in [11].

The function $g(x)$ also has the following property:

Theorem 4.3. The series

$$
g(x)=\sum_{l=1}^{+\infty} \frac{1-2\{l x\}}{l}
$$

converges almost everywhere in $[0,1)$.

The function $g(x)$ was also of interest to L. Báez-Duatre, M. Balazard, B. Landreau and E. Saias. In [1] they studied the function

and proved the following theorem.

$$
A(\lambda):=\int_{0}^{+\infty}\{t\}\{\lambda t\} \frac{d t}{t^{2}} .
$$

Theorem 4.4. Let $\lambda>0$ be such that the series $g(\lambda)$ converges. Then the series $g\left(\frac{1}{\lambda}\right)$ converges too, and we have:

$$
A(\lambda)=\frac{1-\lambda}{2} \log \lambda+\frac{\lambda+1}{2}(\log 2 \pi-\gamma)-g(\lambda)-\lambda g\left(\frac{1}{\lambda}\right) .
$$

Now let us show an important property of $g(x)$, which was proven in [21]. 
Theorem 4.5. There are constants $c_{1}, c_{2}>0$, such that

$$
c_{1} \Gamma(2 k+1) \leq \int_{0}^{1} g(x)^{2 k} d x \leq c_{2} \Gamma(2 k+1)
$$

for all $k \in \mathbb{N}$, where $\Gamma(\cdot)$ stands for the gamma function.

Sketch of a proof. Let us consider the continued fraction expansion of $x$

$$
x=\left[a_{0}(x) ; a_{1}(x), \ldots, a_{k}(x), \ldots\right] .
$$

The $a_{k}(x)$ are obtained via the Gauss map $\alpha$, defined by

$$
\alpha(x)=\left\{\frac{1}{x}\right\}, \alpha_{k}(x)=\alpha\left(\alpha_{k-1}(x)\right), a_{k}(x)=\left\lfloor\frac{1}{\alpha_{k-1}(x)}\right\rfloor .
$$

Definition 4.1. Let $x \in X=(0,1) \backslash \mathbb{Q}$. Let also

$$
\begin{gathered}
\beta_{k}(x)=\alpha_{0}(x) \alpha_{1}(x) \ldots \alpha_{k}(x), \beta_{-1}(x)=1, \\
\gamma_{k}(x)=\beta_{k-1}(x) \log \frac{1}{\alpha_{k}(x)}, \text { where } k \geq 0
\end{gathered}
$$

so that $\gamma_{0}(x)=\log \frac{1}{x}$.

The number $x$ is called a Wilton number if the series

converges.

$$
\sum_{k \geq 0}(-1)^{k} \gamma_{k}(x)
$$

Wilton's function $\mathcal{W}$ is defined by

$$
\mathcal{W}=\sum_{k \geq 0}(-1)^{k} \gamma_{k}(x)
$$

for each Wilton number $x$.

M. Balazard and B. Martin proved in [4] the following proposition:

Proposition 4.1. There is a bounded function $H:(0,1) \rightarrow \mathbb{R}$, which is continuous at every irrational number, such that

$$
g(x)=\mathcal{W}(x)+H(x)
$$

almost everywhere. Also a number $x \in X$ is a Wilton number if and only if $\alpha(x)$ is a Wilton number. In this case, we have:

$$
\mathcal{W}(x)=l(x)-T \mathcal{W}(x)
$$

where

and the operator $T$ is defined by

$$
l(x)=\log \frac{1}{x}
$$

$$
T f(x)=x f(\alpha(x)) .
$$


One can express (40) as

$$
l(x)=(1+T) \mathcal{W}(x) .
$$

The main idea in the evaluation of

$$
\int_{0}^{1} g(x)^{2 k} d x
$$

is to solve the operator equation $(41)$ for $\mathcal{W}(x)$, which is:

$$
\mathcal{W}(x)=(1+T)^{-1} l(x) .
$$

An idea which has long been used in functional analysis for the case when $T$ is a differential operator is to express the right-hand side of (42) as a Neumann series, which is obtained by the geometric series identity, i.e.

$$
(1+T)^{-1}=\sum_{k=0}^{+\infty}(-1)^{k} T^{k}
$$

Thus one can approximate $\mathcal{W}(x)$ by

$$
\mathcal{L}(x, n)=\sum_{k=0}^{n}(-1)^{k}\left(T^{k} l\right)(x) .
$$

Definition 4.2. The measure $m$ is defined by

$$
m(\mathcal{E})=\frac{1}{\log 2} \int_{\mathcal{E}} \frac{d x}{1+x},
$$

where $\mathcal{E}$ is any measurable subset of $(0,1)$.

Proposition 4.2. For $f \in L^{p}$ we have

$$
\int_{0}^{1}\left|T^{n} f(x)\right|^{p} d m(x) \leq g^{(n-1) p} \int_{0}^{1}|f(x)|^{p} d m(x),
$$

where

$$
g=\frac{\sqrt{5}-1}{2}<1 .
$$

Proof. Marmi, Moussa, and Yoccoz, in their paper [22], consider a generalized continued fraction algorithm, depending on a parameter $\alpha$, which becomes the usual continued fraction algorithm for the choice $\alpha=1$. The operator $T_{v}$ is defined in (2.5) of [22] and becomes $T$ for $\alpha=1, v=1$. Then, proposition 4.2 is the content of formulas (2.14), (2.15) of $[22]$.

Using standard techniques of functional analysis one can prove that

$$
\lim _{n \rightarrow \infty} \int_{0}^{1 / 2}\left|\mathcal{L}(x, n)^{L}-\mathcal{W}(x)^{L}\right| d x=0 .
$$


One can eventually prove that

$$
\int_{0}^{1 / 2} \mathcal{L}(x, n)^{2 k} d x=\left(\int_{0}^{1 / 2} l(x)^{2 k}\right)(1+o(1))=\Gamma(2 k+1)(1+o(1)),(k \rightarrow+\infty) .
$$

The order of magnitude of

$$
\int_{0}^{1 / 2} g(x)^{2 k} d x
$$

now follows from (39), by the binomial theorem, since $H(x)$ is a bounded function.

Corollary 4.1. The series

$$
\sum_{k \geq 0} \frac{H_{k}}{(2 k) !} x^{k}
$$

has radius of convergence $\pi^{2}$.

H. Maier and M. Th. Rassias proved in [15] an improvement of Theorem 4.5, by establishing an asymptotic result for the corresponding integral. Namely, they proved the following theorem.

Theorem 4.6. Let

$$
A=\int_{0}^{\infty} \frac{\{t\}^{2}}{t^{2}} d t
$$

and $K \in \mathbb{N}$. There is an absolute constant $C>0$ such that

$$
\int_{0}^{1}|g(x)|^{K} d x=2 e^{-A} \Gamma(K+1)(1+O(\exp (-C K)))
$$

for $K \rightarrow \infty$. $K$.

In [16], they improved this result settling also the general case of arbitrary exponents

Theorem 4.7. Let $K \in \mathbb{R}, K>0$. There is an absolute constant $C>0$ such that

$$
\int_{0}^{1}|g(x)|^{K} d x=\frac{e^{\gamma}}{\pi} \Gamma(K+1)(1+O(\exp (-C K)))
$$

for $K \rightarrow \infty$, where $\gamma$ is the Euler-Mascheroni constant.

\section{Dedekind Sums}

Dedekind sums have applications in many fields of mathematics, especially in number theory. These sums appear in R. Dedekind's study of the function

$$
\eta(s)=e^{\frac{\pi i s}{12}} \prod_{m=1}^{\infty}\left(1-e^{2 \pi i m s}\right),
$$

where $\operatorname{Im} s>0$. 
Definition 5.1. Let $r, b$ be integers, $(r, b)=1, k \geq 1$. Then the Dedekind sum $s\left(\frac{r}{b}\right)$ is defined as follows

$$
s\left(\frac{r}{b}\right):=\sum_{\mu=1}^{b}\left(\left(\frac{r \mu}{b}\right)\right)\left(\left(\frac{\mu}{b}\right)\right),
$$

where $((\cdot))$ is the sawtooth function defined as follows:

$$
((x)):= \begin{cases}x-[x]-\frac{1}{2} & \text { if } x \text { is not an integer }, \\ 0 & \text { if } x \text { is an integer. }\end{cases}
$$

It is a fascinating fact that the Dedekind sum can also be expressed as a sum of cotangent products:

\section{Proposition 5.1.}

$$
s\left(\frac{r}{b}\right)=-\frac{1}{4 b} \sum_{m=1}^{b-1} \cot \left(\frac{\pi m}{b}\right) \cot \left(\frac{\pi m r}{b}\right) .
$$

It is a well-known fact that Dedekind sums satisfy a reciprocity formula:

Theorem 5.1 (Dedekind sums' reciprocity formula).

$$
s\left(\frac{r}{b}\right)+s\left(\frac{b}{r}\right)-\frac{1}{12 r b}=\frac{1}{12}\left(\frac{r}{b}+\frac{b}{r}-3\right) .
$$

We shall not present the proof of Proposition 5.1 and of Theorem 5.1. The interested reader can find these proofs, as well as more fundamental facts concerning Dedekind sums in the now famous book by Rademacher and Grosswald [13].

It is interesting to study the relation between the cotangent sums $c_{0}$ and the Dedekind sums. We've already considered $c_{a}$ for arbitrary $a \in \mathbb{C}$ (see (29)). S. Bettin in [5] proved the very interesting result that $c_{-1}$ is a Dedekind sum up to a constant:

Proposition 5.2. It holds that

$$
s\left(\frac{r}{b}\right)=\frac{1}{2 \pi} c_{-1}\left(\frac{r}{b}\right) .
$$

\section{Sums appearing in the Nyman-Beurling CRiterion for the Riemann Hypothesis CONTAINING THE MÖBIUS FUnCTION}

In a recent paper [17] H. Maier and M. Th. Rassias investigated the following sums (36)

$$
\sum_{n \in I} \mu(n) g\left(\frac{n}{b}\right)
$$

for a suitable interval $I$, where $\mu(n)$ is the Möbius function and the function $g(x)$ is as defined in Definition 2.3.

These sums appear in the study of the integral

$$
\int_{-\infty}^{+\infty}\left|\zeta\left(\frac{1}{2}+i t\right)\right|^{2}\left|D_{N}\left(\frac{1}{2}+i t\right)\right|^{2} \frac{d t}{\frac{1}{4}+t^{2}}
$$


Particularly we could express (50) using formulas (6) and (7) and Proposition 1.1, as follows:

$$
\begin{aligned}
\int_{-\infty}^{+\infty} \mid & \left.\zeta\left(\frac{1}{2}+i t\right)\right|^{2}\left|D_{N}\left(\frac{1}{2}+i t\right)\right|^{2} \frac{d t}{\frac{1}{4}+t^{2}} \\
& =\sum_{1 \leq r, b \leq N} \mu(r) \mu(b)\left(1-\frac{\log r}{\log N}\right)\left(1-\frac{\log b}{\log N}\right) \\
& \times\left[\frac{\log 2 \pi-\gamma}{2}\left(\frac{1}{r}+\frac{1}{b}\right)+\frac{b-r}{2 r b} \log \frac{r}{b}-\frac{\pi}{2 r b}\left(V\left(\frac{r}{b}\right)+V\left(\frac{b}{r}\right)\right)\right]
\end{aligned}
$$

If we expand the last equation, we will obtain the following sum for fixed $b$

which is equal to

$$
\sum_{n \in I} \mu(n)\left(1-\frac{\log n}{\log N}\right) \frac{1}{n} V\left(\frac{n}{b}\right)
$$

$$
\sum_{n \in I} \mu(n)\left(1-\frac{\log n}{\log N}\right) g\left(\frac{n}{b}\right) .
$$

In [17] H. Maier and M. Th. Rassias proved the following result concerning the sums (49):

Theorem 6.1. Let $0 \leq \delta \leq D / 2, b^{2 \delta} \leq B \leq b^{D}$, where $b^{-\delta} \leq \eta \leq 1$. Then there is a positive constant $\beta$ depending only on $\delta$ and $D$, such that

$$
\sum_{B b \leq n \leq(1+\eta) B b} \mu(n) g\left(\frac{n}{b}\right)=O\left((\eta B b)^{1-\beta}\right) .
$$

Finally, the above result was recently improved by the same authors in [18], by proving the following theorem:

Theorem 6.2. Let $D \geq 2$. Let $C$ be the number which is uniquely determined by

$$
C \geq \frac{\sqrt{5}+1}{2}, 2 C-\log C-1-2 \log 2=\frac{1}{2} \log 2 .
$$

Let $v_{0}$ be determined by

$$
v_{0}\left(1-\left(1+2 \log 2\left(C+\frac{\log 2}{2}\right)^{-1}\right)^{-1}+2+\frac{4}{\log 2} C\right)=2 .
$$

Let $z_{0}:=2-\left(2+\frac{4}{\log 2} C\right) v_{0}$. Then for all $\varepsilon>0$ we have

$$
\sum_{b^{D} \leq n<2 b^{D}} \mu(n) g\left(\frac{n}{b}\right) \ll_{\varepsilon} b^{D-z_{0}+\varepsilon} .
$$

\section{REFERENCES}

[1] L. Báez-Duatre, M. Balazard, B. Landreau, E. Saias Etude de l'autocorrelation multiplicative de la fonction 'partie fractionnaire', The Ramanujan Journal, 9, 215-240, 2005

[2] B. Bagchi On Nyman, Beurling and Baez-Duarte's Hilbert space reformulation of the Riemann hypothesis., Proc. Indian Acad. Sci. Math. Sci. 116:2 (2006), 137-146.

[3] R. Balasubramanian, J. Conrey, D. Heath-Brown Asymptoticmeansquare of the product of the Riemann zeta-function and a Dirichlet polynomial, J. Reine Angew. Math. 357 (1985) 161-181 
[4] M. Balazard, B. Martin Sur l'autocorrélation multiplicative de la fonction "partie fractionnaire" et une fonction définie par, J.R. Wilton, arXiv:1305.4395v1

[5] S. Bettin A generalization of Rademacher's reciprocity law, Acta Arithmetica 159 (4), 363-374

[6] S. Bettin On the distribution of a cotangent sum, International Mathematics Research Notices 2015 (21), 11419-11432

[7] S. Bettin, J. Conrey A reciprocity formula for a cotangent sum, International Mathematics Research Notices 2013 (24), 5709-5726

[8] S. Bettin, J. Conrey Period functions and cotangent sums, Algebra \& Number Theory 7 (1), 215-242

[9] S. Bettin, J. Conrey, D. Farmer An optimal choice of Dirichlet polynomials for the Nyman-Beurling criterion, (in memory of Prof. A. A. Karacuba), arXiv:1211.5191

[10] E. Bombieri Problems of the Millenium: The Riemann Hypothesis, Clay Mathematical Institute (2000).

[11] R. de la Bretèche, G. Tenenbaum Series trigonometriques à coefficients arithmetiques, J. Anal. Math. 92 (2004) 1-79.

[12] A. Fokas A novel approach to the Lindel"of hypothesis, arXiv:1708.06607v4 [math.CA] .

[13] E. Grosswald, H. Rademacher Dedekind sums, Mathematical Association of America, (1972), doi:10.5948/UPO9781614440161

[14] M. Ishibashi The value of the Estermann zeta function at $s=0$, Acta Arith. 73(4) (1995) 357-361.

[15] H. Maier, M. Th. Rassias, Asymptotics for moments of certain cotangent sums, Houston Journal of Mathematics, 43(1)(2017), 207-222.

[16] H. Maier, M. Th. Rassias, Asymptotics for moments of certain cotangent sums for arbitrary exponents, Houston Journal of Mathematics, 43(4)(2017), 1235-1249.

[17] H. Maier, M. Th. Rassias, Estimates of sums related to the Nyman-Beurling criterion for the Riemann Hypothesis, Journal of Number Theory, 188(2018), 96-120.

[18] H. Maier, M. Th. Rassias, Explicit estimates of sums related to the Nyman-Beurling criterion for the Riemann Hypothesis, Journal of Functional Analysis (to appear).

[19] H. Maier, M. Th. Rassias Generalizations of a cotangent sum associated to the Estermann zeta function, Communications in Contemporary Mathematics 18 (01), 1550078 (2016).

[20] H. Maier, M. Th. Rassias The maximum of cotangent sums related to Estermann's zeta function in rational numbers in short intervals, Applicable Analysis and Discrete Mathematics, 11(2017), 166-176.

[21] H. Maier, M. Th. Rassias The order of magnitude for moments for certain cotangent sums, J. Math. Anal. Appl. 429 (2015) 576-590

[22] S. Marmi, P. Moussa, J.-C. Yoccoz, The Brjuno functions and their regularity properties, Comm. Math. Phys. 186 (1997) 265-293.

[23] M. Th. Rassias On a cotangent sum related to zeros of the Estermann zeta function, Appl. Math. Comput. 240 (2014) 161-167.

Moscow Institute of Physics and Technology, 141700 Dolgoprudny, Institutskiy Per, D. 9, Russia.

E-mail address: nikita.derevyanko@phystech.edu

National Research University Higher School of Economics, 101000 Moscow, MyasnitSKAYA ULiTsa, D.20, Russia

E-mail address: kdkovalenko@edu.hse.ru 